\title{
Glucocorticoid chronotherapy: a mini-review
}

\begin{abstract}
In this mini-review the chronotherapy with glucocorticoids in rheumatoid arthritis (RA) patients is discussed, as an example of the numerous diseases, showing circadian rhytms in disease activity. Underlying pathophysiological mechanisms are reviewed, explaining the usefullness of glucocorticoid chronotherapy in RA patients. The results of the 2 randomized CAPRA trials, leading to the FDA approval of a modifiedrelease prednisone formula, are discussed, as well as the pricing of these drugs. In a chronopharmacology section it is shown, that some modified drug delivery systems, as MR-prednisone and fast acting meal insulins do not require huge R\&D investments.
\end{abstract}

\author{
Volume 6 Issue 2 - 2018
}

\author{
Michael AB Naafs \\ Endocrinologist, Health Consultant at Naafs, International \\ Health Consultancy, The Netherlands \\ Correspondence: Michael AB Naafs, Dutch Internist, \\ Endocrinologist, Health Consultant at Naafs, International \\ Health Consultancy, Rhodoslaan 20,7577KN, Oldenzaal, The \\ Netherlands, Tel +31681589079, Email naafs.healthconsultancy@ \\ gmail.com,michael.naafs@hotmail.com
}

Received: October 29, 2017 | Published: March 14, 2018

\section{Introduction}

This year's Noble Prize in Physiology or Medicine was awarded to Jeffrey C. Hall, Michael Rosbash and Michael Young, for discovering "key genetic gears"of the body's 24 hour biological clock. Their work identified genes and proteins, that work together in humans and animals to synchronize activities throughout the day and night, regulating sleep patterns, eating habits, blood pressure and hormones, in circadian rythms. ${ }^{1}$ Another Noble Prize in Physiology and Medicine was awarded in 1950 to Philip S. Hench, for his landmark discovery of the benficial effects of glucocorticoids in rheumatoid arthritis (RA). ${ }^{2}$

RA is a typical example of a disease with circadian or diurnal variations in symptoms, as in e.g nocturnal asthma. ${ }^{3}$ RA symptoms, as morning stiffness,joint pain and functional disability are worse in the early morning. ${ }^{4}$ It is now evident, that the morning symptoms in RA, polymyalgia rheumatica (PMR) and ankylosing spondylitis are a result of altered circadian neuroendocrine and inflammatory activities. ${ }^{5}$ Cytokines levels, such as tumor necrosis factor-alpha (TNF-alpha) and interleukin 6 (IL-6) are increased in RA patients at night, in the very early morning hours, whereas they are present at very low levels after noon. Neuroendocrine circadian rythms and "night hormones"as, melatonin and prolactin, as well as the availability of bioenergies at night, are among the triggers of the increased cytokines levels in the early morning hours. ${ }^{6-9}$ The logical next step would be dosing medication for RA patients in the late evening. However nothing is less true. The majority of prescriptions is as a morning dose. More than $90 \%$ of RA patients are taking their medication, when awakening, as symptoms are worse. Glucocoricoids are taken as a single dose then. ${ }^{10}$ In this mini-review, the rational of chronotherapy, its underlyimg mechanisms, and the development of chronopharmacotherapy will be discussed. As implicated above, most of the studies performed on this subject, have been on glucocorticoids in the treatment of RA.

\section{The circadian clock in RA}

The circadian clock is located in the suprachiasmatic nucleus ( $\mathrm{SCN}$ ), a region in the hypothalamus found above the optic chiasm. The $\mathrm{SCN}$ is a central pacemaker collecting light via the retinohypothalamic tract. It also gathers information from peripheral oscillators in organs, cells and tissues. This information is synchronized in the SCN with the aquired retinal information. The peripheral clocks are self-sustaining and can be modified by external factors as temperature. ${ }^{11}$ Liver and lung cells maintained their own rythm in vitro, without light. ${ }^{12-14}$ The endocrine system mediates the dissemination of timing signals from the SCN throughout the body. The "night hormones" cortisol and melatonin are important in the regulation of the immune-inflammatory response, and play a role in the pathogenesis of RA. ${ }^{15}$ The interaction between the circadian clock and the immune-inflammatory system is biderectional. So inflammation can alter cellular of core clock genes, too. ${ }^{16}$

\section{Melatonin}

Melatonin is produced by the pineal gland at night, in a circadian rythm. Serum melatonin is undetectable at daytime, but levels are high at night, in the absence of optical stimulation. ${ }^{17-19}$ The inflammatory cytokines TNF-alpha, IL-1 and IL-6 are secreted from human peripheral blood monocytes in response to melatonin stimulation, and melatonin is detected in RA synovium tissue macrophages and joint fluid. ${ }^{20,21}$ Melatonin levels increase progressively fom 8 p.m to the early morning hours in RA patients, but peak levels are reached 2 hours earlier than in controls, at 2 a.m. The duration of the peak levels, reaching a plateau, was 2-3 hours in patients with RA. This was not observed in controls. Melatonin levels are not correlated with disease activity in RA patients, although early morning melatonin levels are higher in patients with RA of short duration. ${ }^{22}$ These studies suggest, that melatonin treatment might aggrevate RA, but there are no studies, concerning this subject. Antibodies related to RA, such as the $\operatorname{IgA} /$ IgM rheumafactor (RF) and the anti citrullinated protein antibodies (APCA) are also secreted in a circadian manner by B-cells, with a peak in the morning. ${ }^{3}$ Constant disruptions of the circadian clock have been linked to cardiovascular diseases, metabolic syndromes, diabetes and cancer. Night shift work showed an increased risk of RA in women. ${ }^{16}$

\section{Glucocorticoids}

Acute bacterial infections activate the HPA-axis. This leads for a few days to high levels of ACTH(adrenocorticicotrophic hormone) and cortisol. ${ }^{23}$ In chronic inflammatory diseases, such as in RA, cytokines can harm the HPA-axis at any level, resulting in partial adrenal insufficiency. ${ }^{24}$ Cytokines IL-1 beta and TNF-alpha interfere with several steps in steroidgenesis. The circadian rythm of cortisol is not different in healthy controls and in untreated patients with RA. However IL-6 levels are 10 times higher in RA patients and the cytokine circadian rythm is quite different from controls. Thus cortisol secretion is inadequate to the stress of persistent active disease. ${ }^{25}$ In addition, RA synovial cells have an increased activity of 11-beta-hydroxy -steroid dehydrogenase type 2 (11-beta HSD2 ). This results in an increasd degradation of the bioactive cortisol to the biologically inactive cortisone and to a decreased reactivation of cortisone to cortisol. ${ }^{26}$ The clinical and biochemical improvement 
in patients with RA with glucocorticoid (GC) treatment is due to the dampening effect on the pro-inflammatory factors, and the restoration of the steroid milieu. ${ }^{27} \mathrm{GC}$ therapy,often used as a bridge to DMARD (disease modifying anti-rheumatic drugs) therapy, (that needs a few weeks time to work)., can be regarded partly as supplemental therapy in tertiary adrenal insufficiency. The HPA-axis is extendable to the kidney and liver by glucocorticoid metabolism in RA patients. Active cortisol is converted to inactive cortisone by beta-HSD1 in the kidney. The liver is the major organ for converting inactive cortisone to active cortisol by 11-beta HSD2. So dysfunction of the HPA-axis in RA patients is in fact dysfunction of the hepato-hypothalamicpituitary-adrenal-renal axis, by an increased negative feedback loop of active cortisol, and not an adaptation to chronic stress, as supposed before. ${ }^{28}$ Factors that determine adrenal insufficiency are; individual sensitivity, GC dose, duration, formulations of GC therapy and timing of application (circadian). The differences in individual sensitivity are not well understood, yet. CRH (corticotrophin releasing hormone) tests one day after dexamethason administration, showed that a subset of RA patients do not exhibit normal feedback control mechanisms. They had no expected ACTH and cortisol suppression. ${ }^{29}$ The frequency of adrenal suppression increases with increasing GC dosages.$^{30}$ However, low dose GC treatment with 7,5 mg prednisolone daily of RA patients, resulted in some $50 \%$ in blunted ACTH test responses, indicative of adrenal suppression. ${ }^{31}$ Abnormal diurnal rythms of plasma cortisol in patients with RA were found to be related to the total dose of GC given and to the duration of therapy, but not to the mean daily dose or the the daily regimen of therapy. ${ }^{32}$ It has been known for a long time, that splitting the daily dose in several divided doses, strongly increases the risk of adrenal suppression. Whereas endogenous cortisol secretion was not altered with a single dose of $8 \mathrm{mg}$ triamcinolone, application of 4 divided $2 \mathrm{mg}$ doses, resulted in marked suppression of cortisol levels. For that reason GC therapy is generally applied as a single daily dose..$^{33}$

\section{Chronotherapy of GCs}

The time point of application of the single GC dose also plays a role for adrenal suppression.Endogenous cortisol secretion has two peaks; one at 8.a.m and a smaller one at 2.p.m.If exogenous GCs were applied in the evening, this leads to a negative signal on ACTH and endogenous cortisol secretion in the morning. This has been confirmed in several studies. ${ }^{34-38}$ Some RA patients need however dose splitting to control morning stiffness, despite the risk of more HPA-axis suppression. If dose splitting is necessary two third of the dose should be given in the morning(8.a.m.) and one third in the early afternoon (3.p.m.). ${ }^{39}$ Several studies have suggested a greater effect of bedtime or night doses(2.a.m.), in comparison of morning doses of conventional prednisone on morning stiffness..$^{38,40-44}$ HPA-axis suppression was not examined in these studies. Awakening the patient at 2.a.m. was seen as impractical, but conventional prednisone has a pharmacological half-life of only 2 hours. The pharmacokinetics of prednisone have no diurnal rythm. ${ }^{45}$ So the search for a slow-release or modified release preparation started, for covering the cytokine and melatonin peak of RA patients in the early morning. Horizon Pharma, a biopharmaceutical company, developed Rayos, synonyme Lodotra, which is essentially prednisone press- coated as a core in a thick coating. The thick coating and the convex form, instead of the flat form, release the prednisone 4 hours later, allowing them to be taken at bedtime, and being active in the early morning hours. ${ }^{46}$

The efficacy of this modified-release (MR) prednisone formula was investigated in two multicenter, randomized, controlled trials named CAPRA (Circadian Administration of Prednisone in Rheumatoid Arthritis). CAPRA-1 aimed to prove the efficacy and safety of MR prednisone compared to immediate release (IR) prednisone, while
CAPRA-2 focused on MR prednisone, as an additional GC therapy to an existing medication with DMARDs. ${ }^{47}$

CAPRA-1 included 288 patients, already taking IR- prednisone for RA, who were randomized 1:1 to get MR-prednisone in the evening, or continuing IR-prednisone in the morning. The patients have been treated for 12 weeks in both arms. Thereafter there was an open label extension (OLE) of 9 months. Morning stiffness duration was signicantly reduced in the MR-prednisone group, when compared to the IR-prednisone group $(-22,7 \% \mathrm{vs}-0,4 \%)$. This reduction went along with a significant reduction in IL-6 levels, supporting the concept of prednisone chronotherapy. There was no difference in adverse effects (AEs) in both study arms, or in safety profile. ${ }^{48}$ The long-term OLE of CAPRA-1 showed similar results with reduced morning stiffness duration, also for the patients switched from IR to MRprednisone ${ }^{48,49}$ The influence of long term, low dose chronotherapy with MR-prednisone on the HPA- axis was investigated in a subset of 28 patients in the CAPRA- 1 study by CRH -tests. There were no measurable differences in mean cortisol changes after CRH injection, between baseline and the end of the study. There was no indication, that changing treatments from IR-prednisone to MR-prednisone increased the risk of HPA-axis insufficiency, or resulted in detoriation of pre-existing insufficiency. Fifty percent of patients showed a normal response, $37 \%$ showed a suppressed response, and $13 \%$ showed no response, equally divided about both treatment arms.$^{50}$ Clarke et al. ${ }^{51}$ showed an increase in endogenous cortisol secretion after 2 weeks MR-prednisone therapy in patients with active RA, who had received no GCs in the preceding 3 months. Because IL- 6 rise in the morning was suppressed, this might be consistent with a changing relationship between the HPA-axis and the immune system during treatment with MR-prednisone. Further studies are required to test this hypothesis.

CAPRA-2 included 350 RA patients with an existing DMARD therapy and no IR-prednisone medication, 6 weeks prior to the screening. All patients had at least a morning stiffness of 45 minutes. They were randomly assigned to either recieve $5 \mathrm{mg}$ MR-prednisone or placebo in the evening. The primary endpoint was a $20 \%$ improvement in RA signs and symptoms (ACR20 response, Table 1), at the end of the 12-week study. After 12 weeks the MR-prednisone group did not only showed significant improvement in ACR20 response $(48 \%$ vs $29 \% ; \mathrm{p}<0,001)$, but also to ACR50 response $(22 \%$ vs $10 \% ; ; \mathrm{p}<0,006)$. The reduction in mornng stiffness was $(-55 \%$ vs $35 \% ; \mathrm{p}<001) .52$ Studies correlating improved symptom control with the frequency of longterm RA complications are not available.

\section{Table I ACR 20 Response}

A decrease of at least $20 \%$ in both the number of tender and swollen joints along with $20 \%$ reduction in at least three of the following

Patient assessment of disease status

Patients assessment of pain

Physicians's assessment of pain

C-reactive protein level

MR-prednisone is not a new medicine, but at most a new drug drug-design. In Europe Lodotra costs \$ one per tablet, which is still 20 times more than one tablet IR-prednisone. However in the U.S., Rayos is sold for over $\$ 1600$ for 30 tablets ( $\$ 50$ per pill), 350 times more than one single IR-prednisone tablet. This raises the question, whether the use of MR-prednisone is justified and if some patients should set their alarm-clock at 2.a.m. and use IR-prednisone at that time? ${ }^{53}$ 


\section{Chronopharmacology}

Diseases as RA, asthma, diabetes, peptic ulcer, stroke, angina pectoris, myocardial infarction and hypertension follow the body's circadian rythm. Chronopharmacology is aimed to develop drug delivery systems, that can release drugs in a manner, that meets the circadian variation in disease activity. Optimal clinical outcomes can not be achieved, if drug plasma concentrations are constant. Pharmacokinetics can be time depent too. Absorption is mostly better in the morning, than in the evening. This is called chronopharmacokinetics. Furthermore individual sensitivity can vary between women (e.g during mensrual cycles), man and race etnicity and metabolic genomes. ${ }^{54}$ Numerous chronopharmacokinetic studies showed, that the time of drug administration affects drug kinetics. ${ }^{55,56}$ Various technologies to develop time-controlled per-oral drug delivery systems have been studied in recent decades, such as enteric coated systems, layered systems pulse-release systems, sigmoidal release systems and press-coated systems.The MR- prednisone formula can not be called an innovation, as press- coating exists for decades. Press -coating, also known as compresssion coating is relatively simple and cheap, and involves direct compression of the core and the coating, obviating the need for a seperate coating process and the use of coating solutions. ${ }^{57,58}$

A2013 editorial by Barry in the Indian Journal of Pharmacology was titled "Chronotherapies: A hype or future of chronopharmacology?" It was concluded, that chronopharmacology offers a further dimension for research for safer and efficient disease therapy in the future. ${ }^{59}$ Recent examples of chronopharmacology are the fast-acting meal insulins. To the 20 year old insulin aspart, the addition of niacinamide (vitamin B3) increased the speed of insulin absorption. Insulin FIASP (Faster-Acting Insulin Aspart) is sold in the UK fort he same price as for Novo Rapid Aspart, while definite pricing in the U.S is not known yet. Insulin FIASP is a useful expansion of the insulin assortiment. It is not a big innovation, requiring billions of research dollars as is Royas, Lotendra neither at all. Anyway Insulin FIASP will not have the exorbitant prices of Royas and Lotendra. Indeed chronopharmacology can become the wrong hype in this way. Nevertheless, "Mind the Time"was the title of a recent PHD thesis at Leiden University, by Laura Kervezee. ${ }^{62}$

\section{Conclusion}

Rheumatoid arthritis is a typical example of a disease with a circadian activity,showing serious morning stifnness. Night hormones as melatonin and cortisol play by their interaction with the immune-cytokine system a role in the pathogenesis of RA. There is a bidirectional interaction between the circadion clock, located in the supra chiasmatic nucleus ( $\mathrm{SCN}$ ) and the immune system. The endocrine system mediates the dissemination of timing signals. Chronic inflammation can alter cell or core clock genes. Melatonin stimulates cytokine production of IL-6 and TNF -alpha in blood monocytes and synovium macrophages in RA patients in a circadian rythm.Cytokines can harm the HPA-axis at any level, resulting in tertiary adrenal insufficiency in half of the RA patients. The early morning rise in IL-6 secretion can be dampened by a modifiedrelease prednisone formula, MR-prednisone, when administered at the evening. In 2 randomized trials CAPRA-1 and CAPRA-2) MR-prednisone taken at the evening reduced morning stiffness significantly, compared with immediate release (IR) prednisone taken in the morning. MR-prednisone decreased the early IL-6 morning rise significantly over IR-prednisone and relieved RA symptoms in combnation with DMARDs, compared to DAMDR monotherapy. In addition MR-prednisone might have a favourable effect on the HPA-axis by increasing endogenous cortisol secretion in RA patients, without prior GC medication. The price of MR-prednisone is unacceptable high, however. Therefore it should be reserved for those RA patients,in whom morning stiffness can not be controlled. As a lot of diseases, such as diabetes, RA, asthma, hypertension, myocardial infarction, stroke show circadian rythms, chronopharmacology should have a bright future.

\section{Aknowledgement}

None.

\section{Conflic of interest}

None.

\section{References}

1. The Noble Prize in Physiology or Medicine 2017. Nature.com; Collection; 2017.

2. Broadway D,Wilder R. Philip Hench-Cortisone and Rheumatoid Arthritis, 1948. Brain Immune Trends; 2009.

3. Straub RH, Cutolo M. Circadian rythms in rheumatoid arthritis:implications for pathphysiology and therapeutic management. Arthritis Rheum. 2007;56(2):399-408.

4. Spies CM, Cutolo M, Straub RH, et al. More night than day: circadian rythms in polymyalgia rheumatica and ankylosing spondylitis. $J$ Rheumatol. 2010;37(5):894-899.

5. Straub RH, Bijlsma JW, Masi A, et al. Role of neuroendocrine and neuroimmune mechanisms in chronic inflammatory rheumatic diseasesthe 10 year update. Semin Arthritis Rheum. 2013;43(3):392-404.

6. Cutolo M, Seriolo B, Craviotto C, et al. Circadian Rhytms in RA. Ann Rheum Dis. 2003;62(7):593-596.

7. Buttgereit F, Smolen JS, Coogan AN. Clocking in: chronobiology in rheumatoid arthritis. Nat Rev Rheumatol. 2015;11(6):349-356.

8. Cutolo M. Rheumatoid Arthritis: circadian and circannual rythms in RA. Nat Rev Rheumatol. 2011;7(9):500-502.

9. Straub RH, Cutolo M, Buttgereit F, et al. Energy regulation and neuroendocrine immune control in chronic inflammatory diseases. J Int Med. 2010;276(6):543-560.

10. Kirwan J. Targeting the time of the day for glucocorticoid delivery in rheumatoid arthritis. Int J Clin Rheumatol. 2011;6(3):273-279.

11. Albrecht U. Timing to perfection: the biology of central and peripheral circadian clocks. Neuron. 2012;74(2):246-260.

12. Yoshida K, Hashimoto T, Sakai Y, et al. Circadian rythm and joint stiffness/destruction in rheumatoid arthritis. Int $J$ Clin Rheumatol. 2015;10(5):335-344.

13. Reppert SM,Weaver DR. Coordination of circadian timing in mammals. Nature. 2002;418(9601):935-941.

14. Okamura H. Clock genes in cell clocks: roles, actions and mysteries. $J$ Biol Rythms. 2004;19(5):388-399.

15. Coogan AN, Wyse CA. Neuroimmunology of the circadian clock. Brain Res. 2008;1252:104-112.

16. Wang XS, Armstrong ME, Cairns B, et al. Shift work and chronic disease; the epidemiological evidence. Occup Med. 2011;61(2):78-89.

17. Cardinali DP, Pever P. Basic aspects of melatonin action. Sleep Med Rev. 1998;2(3):175-190. 
18. Chuang JI, Chen SS, Lin MT. Melatonin decreases brain serotonin release, arterial pressure and heart rates in rats. Pharmacology 1993;47(2):91-97.

19. Gilbert SS, van den Heuvel CJ, Dawson D, et al. Daytime melatonin and temazepam in young adult humans; equivalent effects on sleep latency and body temperature. J Physiol.1999;514(Pt 3):905-914.

20. Gibbs JE, Ray DW. The role of the circadian clock in rheumatoid arthriis. Arthritis Res Ther. 2013;15(1):205.

21. Cutolo M, Maestreoni GJ, Otsa K, et al. Circadian melatonin and cortisol levels in rheumatoid arthritis patients in winter time; a north and south Europe comparison. Ann Rheum Dis. 2005;64(2):212-216.

22. Afkhamizadeh M, Sahebari M, Seyed-Hoseini SR. Morning melatonin serum values do not correlate with disease activity in rheumatoid arthritis: a cross sectional study. Rheumatol Int. 2014;34(8):1145-1151.

23. Kanczkowski W, Sue M, Zacharowski K, et al. The role of adrena gland microenvironment in the HPA axis function and dysfunction during sepsis. Mol Cell Endocrinol. 2015;408:241-248.

24. Annetta M, Maviglia R, Proietti R, et al. Use of corticosteroids in critically ill septic patients: a review of mechanisms of adrenal insufficiency in sepsis and treatment. Curr Drug Targets. 2009;10(9):887-894.

25. Crofford LJ, Kalogeras KT, Mastorakas G, et al. Circadian relationships between interleukin (IL)-6 and hypothalamic-pituitary-adrenal axis hormones; failure of IL-6 to cause sustained hypercortisolism in patients with early untreated rheumatoid arthritis. $J$ Clin Endocrinol Metab. 1997;82(4):1279-1283.

26. Schmidt M,Weidler C, Naumann H, et al. Reduced capacity for the reactivation of glucocorticoids in rheumatoid arthritis of synovial cells: Possible role of the sympathetic nervous system? Arthritis Rheum 2005;52(6):1711-1720.

27. Cutola M, Foppiani L, Minuto F. Hypothalamic-pituitary-adrenal axis impairment in the pathogenesis of rheumatoid arthritis and polymyalgia rheumatica. J Endocrinol Invest. 2002;25(10 Suppl):19-23.

28. Edwards C. Sixty years after Hench:corticosteroids and chronic inflammatory disease. J Clin Endocrinol Metab. 2012;97(5):14431451

29. Harbuz MS, Korendowych E, Jessop DS, et al. Hypothalamic-pituitaryadrenal axis dysregulation in patients with rheumatoid arthritis after the dexamethason/corticotrophin releasing factor test. $J$ Endocrinol. 2003;178(1):55-60.

30. Klinefelter HF,Winkenwerder WL, Bledsoe T. Single daily dose prednisone therapy. JAMA. 1979;241(25):2721-2723.

31. Kirwan JR, Hickley SH, Hallgren R, et al. The effect of therapeutic glucocorticoids on the adrenal response in a randomized controlled trial in patients with rheumatoid arthritis.Arthritis Rheum. 2006;54(5):14151421.

32. Jasani MK, Diver MJ, Belt AM, et al. Some clinical observations on the diurnal rythm of plasma cortisol (11-OHCS) in patients with rheumatoid arthritis treated with oral corticosteroid drugs. Curr Med Res Opin. 1974;2(7):373-386.

33. Grant SD, Forsham PH, Di Raimondo VC. Suppression of 17-hydroxycorticosteroids in plasma and urine by single or divided doses of triamcinolone. N Engl J Med. 1965;273(21):1115-1118.

34. Haus E, Suckett-Lundeen L, Smolensky MH. Rheumatoid Arthritis circadian rythms in disease activity,signs and symptoms and rationals for chronotherapy with corticosteroids and other medications. Bull NYU Hosp J Dis. 2012;70(Suppl 1):3-10.
35. Angeli A. Circadian ACTH adrenal rythm in man. Chronobiologica. 1974;1(Suppl):253-270

36. Nichols T, Nugent CA, Tyler FH. Diurnal variation in suppression of adrenal function by glucocorticoids. $J$ Clin Endocrinol Metab. 1965;25:343-349.

37. Di Raimondo VC, Forsham PH. Some clinical implications of the spontaneous diurnal variation in adrenal cortical secretory activity. $\mathrm{Am}$ J Med. 1956;21(3):321-323.

38. Deandrade JR, McCormick JH, Hill AG. Small doses of prednisolone in the management of rheumatoid arthritis. Ann Rheum Dis. 1964;23:158162 .

39. Reinberg A, Guillet P, Gervais P. One month chronocorticotherapy (Dutimelan 8-15 mite):Control of the asthmatic condition without adrenal suppression and cicadian rythm alteration. Chronobiologica. 1977;4(4):295-212.

40. Arvidson NG, Gudbjornsson B, Larsson A. The timing of glucocorticoid administration in rheumatoid arthritis. Ann Rheum Dis. 1997;56(1):2731.

41. De Silva M, BinderA, Hazleman BL. The timing of prednisolone dosage and its effect on morning stiffness in rheumatoid arthritis. Ann Rheum Dis. 1984;43(6):790-793.

42. Owlia MB, Mehrpoor G, Modares-Mosadegh M. Bedtime single dose prednisolone in clinically stable rheumatoid arthritis patients. ISRN Pharmacol. 2012:637204.

43. Karatay S, Senel K, Ugar M, et al. The timing of low dose glucocorticoid therapy in the treatment of rheumatoid arthritis. Pain Clinic. 2002;13:305-312.

44. Kowanko IC, Pownell R, Knapp MS. Circadian variations in the signs and symptoms of rheumatoid arthritis and in the therapeutic effectiveness of flurbiprofen at different times of the day. $\mathrm{Br} J \mathrm{Clin}$ Pharmacol. 1981;11(5):477-484.

45. McAllister WA, Mitchell DM, Collins JV. Prednisolone pharmacokinetics compared between day and night in asthmatic and normal subjects. Br J Clin Pharmacol. 1981;11(3):303-304.

46. Rohan S, Parul K, Farhutjan S. Chronotherapeutical approach: Circadian rythm in human and its role in occurence and severity of diseases. Int $J$ Pharm Tech Res. 2012;4(2):765-777.

47. Krasselt M, Baehrwald C. Efficacy and safety of modified-release prednisolone in patients with rheumatoid arthritis. Drug Des Devel Ther. 2016;10:1047-1058.

48. Buttgereit F, Doering G, Schaeffler A, et al. Targeting pathophysiological rythms;prednisone chronotherapy shows sustained efficacy in rheumatoid arthritis. Ann Rheum Dis. 2010;69(7):1275-1280.

49. Buttgereit F, Doering G, Schaeffler A, et al. Efficacy of modifiedrelease versus standard prednisone to reduce morning stiffness of the joints in rheumatoid arthritis (CAPRA-1); a double blind,randomized, controlled trial. Lancet. 2008;371(9608):205-214.

50. Allen R, Doring G, Cutola M, et al. Hypothalamic-pituitary-adrenal axis function in patients with rheumatoid arthritis treated with nighttime-release prednisone. J Rheumatol. 2010;37:2025-2031.

51. Clarke L, Jessop DS, Hunt LP, et al. Alleviation of morning joint stiffness by low-dose prednisone in rheumatoid arthritis is associated with circadian changes in IL-6 and cortisol. Int J Clin Rheumatol. 2011;6(2):241-249. 
52. Buttgereit F, Mehta D, Kirwan I, et al. Low dose prednisone chronotherapy for rheumatoid arthritis; a randomized clinical trial .(CAPRA-2). Ann Rheum Dis. 2013;72(2):204-210.

53. Conn DL. Is the Availability of Delayed Release Preenisone an Important Clinical Advance? Arthritis Care \& Research. 2016;68(3):412-413.

54. Sajan J, Cinu TA, Chacko AJ. Chronotherapeutics and Chronotropic Drug Delivery Systems. Trop J Pharmaceutical Research. 2009;8(5):467-475.

55. Lemmer B. Circadian rythms and drug delivery. $J$ Contr Release. 1991;18:63-74.

56. Lemmer B, Bruguolle B. Chronokinetics:are they clinically relevant? Clin Pharmacokinet. 1994;26:419-427.

57. Gazziniga A, Sangall M, Gorderano F. Oral chronotropic drug delivery systems: Achievement of time and/or site specificity. Eur $J$ Biopharm. 1994;40:246-250.
58. Matsuo M, Nakamura C,Animoni K. Evaluation ofhydroxyethylcellulose as a hydrophyllic availaible material for delayed-release tablets. Chem Pharm Bull. 1995;43(2):311-314.

59. Barry LK. Chronotherapeutics: A hype or a future of chronopharmacology? Indian J Pharmacol. 2013;45(6):545-546.

60. Yough SS, Singh SP, Singh Y, et al. Chronopharmacology:Recent Advancement in the Treatment of Diabetes Mellitus through Chronotherapy. Ijppr Human. 2017;9(2):87-99.

61. Russel-Jones D, Bode BW, de Block C, et al. Fast-Acting Insulin Aspart Improves Glycaemic Control in Basal Bolus Treatment for Type 1 Diabetes:Results of a 26 Week Multicenter, Active-Controlled, Treat to-Target, Randomized, Parallel-Group Trial (onset-1). Diabetes Care. 2017;40(7):943-950.

62. Kervezee L. Mind the time-24 hour rythms in drug exposure and effect. Thesis, Leiden University; 2017. 